\title{
Résumé de la mise à jour de la Déclaration du Comité consultatif national de l'immunisation (CCNI) sur l'usage recommandé du vaccin contre I'hépatite $A$
}

\author{
Henry $\mathrm{B}^{1}$, Baclic $\mathrm{O}^{2}$ au nom du Comité consultatif national de l'immunisation $(\mathrm{CCNI})^{\star}$
}

\section{Résumé}

Contexte : La gravité de I'hépatite A augmente avec l'âge. Les enfants de moins de six ans sont généralement asymptomatiques ou présentent les signes d'une hépatite $A$ bénigne sans ictère. Ils représentent une source d'infection importante, en particulier pour les contacts familiaux et les autres contacts étroits. Chez les enfants plus âgés et les adultes, I'hépatite A est généralement symptomatique. Les personnes plus âgées et les sujets souffrant d'une maladie chronique du foie ou d'affections liées à l'immunodépression sont exposés à un risque accru d'évolution vers une insuffisance hépatique fulminante entraînant la mort. L'utilisation d'un vaccin contre l'hépatite $A$ est recommandée pour l'immunisation préexposition des personnes présentant un risque d'infection accru ou une hépatite $A$ grave, ainsi que dans les 14 jours suivant l'exposition à l'hépatite $A$ pour les contacts familiaux et des contacts étroits réceptifs des cas avérés ou présumés d'hépatite $A$, des collègues et clients des personnes infectées qui manipulent des aliments, et lorsque l'hépatite $A$ touche les employés et les participants des centres de garde d'enfants ou des écoles maternelles. Le Comité consultatif national de l'immunisation (CCNI) du Canada a déjà recommandé la vaccination contre l'hépatite A chez les personnes d'un an et plus.

\begin{abstract}
Objectifs : Formuler des recommandations sur l'utilisation du vaccin contre I'hépatite A chez les nourrissons de moins d'un an et clarifier les recommandations post-exposition dans les cas d'immunoglobulines humaines (lg).
\end{abstract}

Méthodologie : Le groupe de travail sur l'hépatite A du CCNI a effectué des examens de la documentation et a passé en revue les données fournies par le fabricant du vaccin contre l'hépatite A sur le sujet de la prophylaxie post-exposition. Toutes les données probantes ont été évaluées et signalées dans les tableaux de données probantes. Une synthèse des connaissances a été réalisée, et le $\mathrm{CCNI}$ a approuvé des recommandations précises fondées sur des données probantes, clarifiant le raisonnement et les considérations pertinentes.

Résultats : Aucune étude sur l'efficacité potentielle et réelle du vaccin à composant anti-hépatite $A$ chez les enfants de six mois à moins de 12 mois n'a été trouvée lors de la recherche documentaire. L'administration de deux doses de vaccins à composant anti-hépatite a été établie comme sécuritaire et immunogène chez les nourrissons âgés de six mois à 12 mois. Les données disponibles concernant l'immunogénicité du vaccin contre l'hépatite A chez les adultes de plus de 40 ans étaient limitées.

Conclusion : Il y a maintenant de nouvelles recommandations du CCNI sur le vaccin contre l'hépatite A et l'utilisation post-exposition des immunoglobulines humaines.

\author{
Affiliations \\ ${ }^{1}$ Président du groupe de travail \\ sur l'hépatite du CCNI, Victoria \\ (Colombie-Britannique) \\ ${ }^{2}$ Centre de l'immunisation \\ et des maladies respiratoires \\ infectieuses, Agence de la santé \\ publique du Canada, Ottawa \\ (Ontario)
}

*Correspondance : naci-ccni@ phac-aspc.gc.ca
Citation proposée : Henry B, Baclic O au nom du Comité consultatif national de l'immunisation (CCNI). Resumé de la mise à jour de la déclaration du Comité consultatif national de l'immunisation (CCNI) sur l'usage recommandé du vaccin contre I'hépatite A. Relevé des maladies transmissibles au Canada 2016;42:215-8. https://doi.org/10.14745/ccdr.v42i09a07f

\section{Introduction}

La gravité de I'hépatite A augmente avec l'âge. Les enfants de moins de six ans sont souvent asymptomatiques ou présentent des cas légers de la maladie. Chez les enfants plus âgés et les adultes, I'hépatite $A$ est généralement symptomatique. Les personnes plus âgées et les sujets souffrant d'une maladie chronique du foie ou d'affections liées à l'immunodépression sont exposés à un risque accru d'évolution vers une insuffisance hépatique fulminante entraînant la mort. L'utilisation d'un vaccin contre l'hépatite A est recommandée pour l'immunisation préexposition des personnes présentant un risque d'infection 
accru ou une hépatite A grave, ainsi que dans les 14 jours suivant l'exposition à l'hépatite A pour les contacts familiaux et des contacts étroits réceptifs des cas avérés ou présumés d'hépatite $A$, des collègues et clients des personnes infectées qui manipulent des aliments, et lorsque l'hépatite $A$ touche les employés et les participants des centres de garde d'enfants ou des écoles maternelles.

Le Comité consultatif national de l'immunisation (CCNI) du Canada a déjà recommandé la vaccination préexposition contre I'hépatite A chez les personnes d'un an et plus. Le groupe de travail sur l'hépatite du CCNI a récemment terminé son travail sur l'élaboration de recommandations quant à l'utilisation du vaccin contre I'hépatite A chez les nourrissons de moins d'un an et afin de clarifier les recommandations sur l'utilisation post-exposition des immunoglobulines humaines (lg). Pour cela, il a effectué des examens de la documentation et a passé en revue les données fournies par le fabricant du vaccin contre l'hépatite A sur le sujet de la prophylaxie post-exposition. Toutes les données probantes ont été évaluées et signalées dans les tableaux de données probantes. Une synthèse des connaissances a été réalisée, et le CCNI a approuvé des recommandations précises fondées sur des données probantes, clarifiant le raisonnement et les considérations pertinentes.

L'examen de la documentation sur l'utilisation du vaccin contre I'hépatite $A$ et les recommandations actuelles sur le vaccin contre I'hépatite A sont publiés dans la version intégrale de la mise à jour de la déclaration du CCNI (1) et le chapitre relatif à I'hépatite A du Guide canadien d'immunisation (2). Pour résumer, aucune étude sur l'efficacité potentielle et réelle du vaccin à composant anti-hépatite $A$ chez les enfants de 6 mois à moins de 12 mois n'a été trouvée lors de la recherche documentaire. L'administration de deux doses de vaccins à composant anti-hépatite a été établie comme sécuritaire et immunogène chez les nourrissons âgés de 6 mois à 12 mois. Les données disponibles concernant l'immunogénicité du vaccin contre I'hépatite A chez les adultes de plus de 40 ans étaient limitées. Les recommandations clés sont résumées ci-dessous.

\section{Recommandations de 2016 du CCNI sur le vaccin contre l'hépatite $A$ et l'utilisation post- exposition de l'immunoglobuline}

Recommandation 1 : Le vaccin contre I'hépatite A peut être administré à des nourrissons d'au moins six mois qui présentent un risque d'infection accru ou une hépatite $A$ grave. (Recommandation du CCNI de catégorie B)

Les nourrissons souffrant d'une maladie sous-jacente du foie dont la cause est idiopathique, métabolique, infectieuse ou cholostatique peuvent faire partie des nourrissons présentant un risque accru d'infection grave à l'hépatite A. Les nourrissons nés au Canada qui voyagent dans des pays où l'hépatite A est endémique, y compris les enfants nés au Canada de néo-Canadiens retournant dans leur pays d'origine pour rendre visite à des amis ou de la famille, peuvent être exposés à un risque accru d'infection à l'hépatite $A$.

Recommandation 2 : Le vaccin contre l'hépatite A peut être administré à des nourrissons d'au moins six mois qui sont des contacts familiaux de sujets présentant un risque d'infection accru ou une hépatite A grave. (Recommandation du CCNI de catégorie B)

Recommandation 3 : Pour la prophylaxie post-exposition, à moins qu'il soit contre-indiqué ou indisponible, le vaccin contre l'hépatite $A$ est recommandé de préférence aux immunoglobulines pour les sujets en santé de six mois et plus. (Recommandation du CCNI de catégorie B)

Étant donné que la teneur en anticorps de I'hépatite A dans les immunoglobulines est censée diminuer avec le temps à cause des titres d'anticorps plus faibles au sein de la population (dus à des taux d'infection naturelle plus faibles), et que le profil d'innocuité du vaccin à composant anti-hépatite $A$ inactivé est excellent, l'immunisation est préférable à l'administration d'un produit dérivé du sang.

Recommandation 4 : L'immunisation avec le vaccin contre I'hépatite A peut être envisagée pour tous les sujets recevant, à plusieurs reprises, des facteurs de coagulation dérivés du plasma. (Recommandation du CCNI de catégorie I)

Le traitement par solvant-détergent utilisé pour préparer les concentrés de facteurs de coagulation dérivés du plasma n'inactive pas le virus de l'hépatite $A$ de manière fiable. Cependant, il n'y a pas eu dans le passé de preuve de la transmission de l'hépatite A par les facteurs de coagulation dérivés du plasma au Canada, et le risque de transmission par transfusion est très faible puisque tout le plasma utilisé subit un test de dépistage de l'hépatite A. À cause d'une possibilité théorique d'infection, l'immunisation des sujets recevant d'importantes quantités de facteurs de coagulation dérivés du plasma doit être prise en compte. Au Canada, les monographies des produits dérivés du plasma utilisés dans le traitement des maladies nécessitant le remplacement de facteurs de coagulation comprennent des recommandations pour l'immunisation avec le vaccin contre l'hépatite $A$.

Recommandation 5 : Pour la prophylaxie post-exposition dans les 14 jours suivant l'exposition d'adultes réceptifs de 60 ans et plus qui sont des contacts familiaux ou étroits d'un cas, les immunoglobulines peuvent être administrées en plus du vaccin contre I'hépatite A. (Recommandation du CCNI de catégorie I)

Les sujets qui n'ont pas d'antécédents de maladie ou vaccinaux sont réceptifs aux infections par le virus de l'hépatite $A$. Les données probantes laissent entendre une baisse de la réponse immunogène au vaccin contre l'hépatite $A$, ainsi qu'une hausse des hospitalisations et des taux de létalité liés à l'hépatite $A$, en fonction du vieillissement. Toutefois, en raison de l'incertitude considérable concernant la valeur ajoutée d'une immunisation passive sur l'issue de la maladie (incluant la teneur en anticorps de l'hépatite A dans les immunoglobulines), de la prévalence élevée des anticorps de l'hépatite A dans les groupes d'âge plus avancé et du peu de cas de complications liées aux infections par le virus de l'hépatite A chez les sujets de 60 ans et plus, la décision d'inclure des immunoglobulines pour la prophylaxie post-exposition doit être prise au cas par cas. Étant donné le manque de données appuyant les avantages des immunoglobulines lorsqu'elles sont administrées après les 14 jours, il n'existe aucune recommandation pour leur utilisation après cette période. La prophylaxie post-exposition avec vaccin 
uniquement est recommandée lors des interventions en cas d'éclosion.

Recommandation 6 : Pour la prophylaxie post-exposition de sujets réceptifs souffrant d'une maladie chronique du foie, les immunoglobulines doivent être administrées dans les 14 jours suivant l'exposition, en plus du vaccin contre l'hépatite $A$. (Recommandation du CCNI de catégorie B)

En raison du risque de maladie sévère et d'une réaction immunitaire sous-optimale au vaccin contre l'hépatite A chez les sujets immunocompromis et atteints d'une maladie chronique du foie, les immunoglobulines sont recommandées pour fournir une protection immédiate contre une infection par le virus de I'hépatite $A$ jusqu'à ce qu'une réponse active au vaccin soit produite. Étant donné le manque de données appuyant les avantages des immunoglobulines lorsqu'elles sont administrées après les 14 jours, il n'existe aucune recommandation pour leur utilisation après cette période.

\section{Financement}

Les travaux du CCNI sont appuyés par l'Agence de la santé publique du Canada.

\section{Conflit d'intérêts}

Aucun.

\section{Références}

1. Comité consultatif national de l'immunisation (CCNI). Mise à jour sur les recommandations concernant l'utilisation du vaccin contre I'hépatite A. Ottawa ON: PHAC; 2016. http:// www.canadiensensante.gc.ca/publications/healthy-living-viesaine/hepatitis-a-vaccine-update-recommended-use-2016mise-a-jour-recommandations-hepatite-a-vaccin/index-fra. php.

2. Comité consultatif national de l'immunisation (CCNI). Guide canadien d'immunisation: Partie 4 : Vaccin contre l'hépatite A. Ottawa ON: ASPC; 2016. [Date de modification : août 2016]. http://www.canadiensensante.gc.ca/publications/ healthy-living-vie-saine/4-canadian-immunization-guidecanadien-immunisation/index-fra.php?page $=6$.

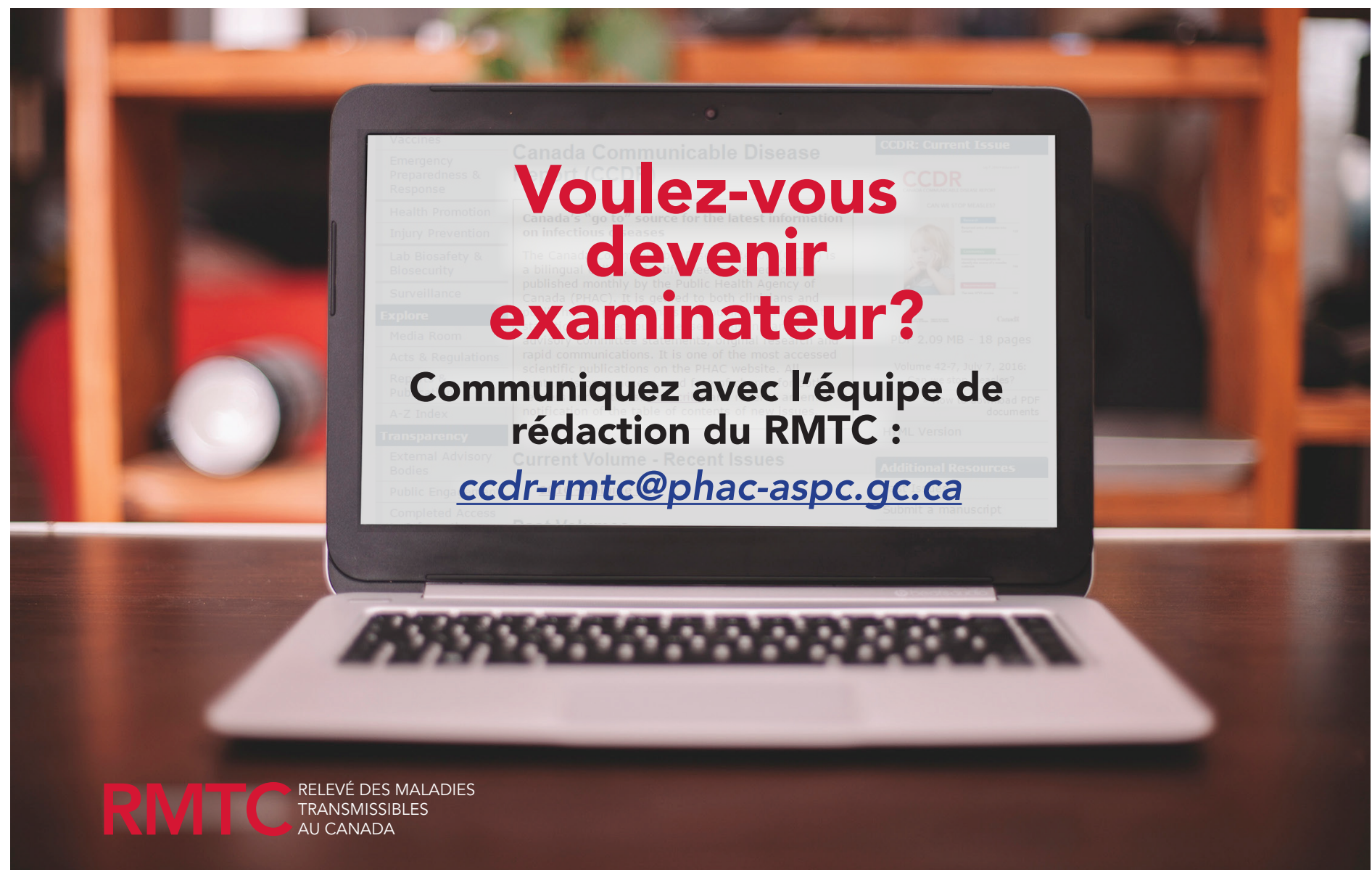

